\title{
Safety and efficacy of a novel iopromide-based paclitaxel-eluting balloon following bare metal stent implantation in rabbit aorta abdominalis
}

\author{
Zhengbin Zhu ${ }^{\text {a,b,* }}$, Hui Han ${ }^{\text {b,* }}$, Jinzhou Zhu ${ }^{\text {a,b,* }}$, Jing Zhang ${ }^{c}$, Run Du ${ }^{\text {a }}$, Jingwei Ni ${ }^{\text {a }}$, \\ Chen Ying ${ }^{\mathrm{a}}$, Xuanqi An ${ }^{\mathrm{a}}$ and Ruiyan Zhang ${ }^{\mathrm{a}, \mathrm{b}, * *}$ \\ a Department of Cardiology, Rui Jin Hospital, School of Medicine, Shanghai Jiao Tong University, \\ Shanghai, China \\ ${ }^{\mathrm{b}}$ Institute of Cardiovascular Diseases, School of Medicine, Shanghai Jiao Tong University, Shanghai, \\ China \\ ${ }^{\mathrm{c}}$ Shanghai Pharmaceuticals Holding Co. Ltd, Central Research Institute, Shanghai, China
}

Received 13 April 2015

Accepted 3 August 2015

\begin{abstract}
.
BACKGROUND: Drug-eluting balloons (DEB) may be promising technology for treating atherosclerotic arterial disease. In fact, several DEBs have been clinically available for the treatment of coronary in-stent restenosis (ISR), de novo coronary lesions, and peripheral artery disease.

OBJECTIVE: We sought to elucidate the mechanism of action and in vivo safety and efficacy of a novel iopromide-based paclitaxel-eluting balloon.

METHODS: In vitro cytotoxicity of a novel DEB on human umbilical vein endothelial cells (HUVECs) and in vivo pharmacokinetics of DEB in a rabbit aorta abdominalis were assessed. Then, bare metal stents (BMS) were implanted at both the proximal and distal sites of the rabbit aorta abdominalis. Stented vascular segments were immediately dilated with a bare balloon (control group) or the DEB (DEB group) randomly. Histological evaluation was performed in all treated segments at 28 days. Because paclitaxel is a tubulin-disrupting agent that binds preferentially to $\beta$-tubulin, we measured $\beta$-tubulin expression in aortal stent specimens via immunohistochemistry.

RESULTS: We observed that DEB was compatible and could reduce neointimal hyperplasia compared with the bare balloon. Meanwhile, immunohistochemistry revealed that $\beta$-tubulin expression in the DEB group increased compared with the control group, indirectly suggesting successful uptake of paclitaxel by vessel walls after DEB dilation.

CONCLUSIONS: The novel DEB is safe and has a favorable vascular healing response on neointimal hyperplasia.

Keywords: Neointimal hyperplasia, paclitaxel-eluting balloon, pharmacokinetics

\footnotetext{
*Zhengbin Zhu, Hui Han and Jinzhou Zhu contributed equally in this study.

** Corresponding author: Ruiyan Zhang, Department of Cardiology, Rui Jin Hospital, School of Medicine, Shanghai Jiao Tong University, 197 Ruijin Road II, Shanghai, 200025, China. Tel.: +86 21 64370045; Fax: +86 21 64457177; E-mail: rjzhangruiyan@aliyun.com.
} 


\section{Introduction}

Vascular stenosis and occlusion caused by atherosclerosis is a leading cause of death globally and these are initiated by coronary artery disease (CAD), stroke, and peripheral artery disease [1]. As a standard therapy, percutaneous transluminal angioplasty (PTA) has been upgraded from simple balloon angioplasty to bare metal stent (BMS) implantation, and on to drug-eluting stent (DES) treatment [2]. Early vessel recoil and subsequent neointimal hyperplasia caused by arterial remodeling still causes restenosis, however, and this limits the long-term clinical outcomes of balloon angioplasty [3]. These limitations were thought to have been largely eliminated with the introduction of BMS [4]. However, BMS stimulated greater neointimal and produced a new problem: in-stent restenosis (ISR) [5]. DES were then developed and were reported to reduce neointimal hyperplasia [6,7] and this decreased ISR to $\sim 5-10 \%$ after treating coronary lesions [8]. Even so, restenosis rates are unacceptably high and the small but unpredictable risk of very late stent thrombosis may still occur due to hypersensitivity reactions to the stent polymer coating $[9,10]$.

Thus, novel strategies to ameliorate neointimal hyperplasia and stent thrombosis are needed. The goal of using PTA without leaving behind a permanent device, led to the development of drug eluting balloons (DEB) [11]. First experiments with DEBS included paclitaxel-coated balloons (PCB) in pigs [12,13], and soon DEB was seen as an alternative treatment to plain balloon angioplasty and stent implantation. Studies with promising long-term results with DEB for treating coronary ISR compared with uncoated balloons and paclitaxel-coated stents were published [14-16] and reports of DEB treatment of lesions in small coronary arteries followed [17,18].

We sought to assess the blood compatibility of a newly designed iopromide-based paclitaxel-eluting balloon in vitro, and evaluate the efficacy of the DEB by measuring vessel neointimal hyperplasia in a rabbit aorta abdominalis model.

\section{Materials and methods}

\subsection{Device description}

DEB catheters are single-use hydrophilic coated with polyvinylpyrrolidone (PVP). Balloons were $3.0 \times 20 \mathrm{~mm}$ coated with a paclitaxel-iopromide formulation $\left(3 \mu \mathrm{g} / \mathrm{mm}^{2}\right.$ of balloon surface $)$ using a proprietary dipping process that deposited the formulation preferentially in the folds of the balloon.

\subsection{Cytotoxicity testing}

Human umbilical vein endothelial cells (HUVECs) were seeded at low density (30,000 cells per 12well culture plates) and cultured in M200 supplemented with $10 \%$ fetal bovine serum (FBS) and growth factors for $24 \mathrm{~h}$. Cells were then co-incubated with an iopromide-coated balloon without paclitaxel $(n=6)$ for 2 days. HUVEC cultures were incubated with a bare balloon as controls $(n=6)$. After the incubation period, $50 \mu \mathrm{l}$ of cell proliferation reagent, WST-1 (Roche Applied Science, Mannheim, Germany) was added to each well. After $4 \mathrm{~h}$ of WST-1 addition, absorbance at $450 \mathrm{~nm}$ was measured via an enzyme-linked immunosorbent assay (ELISA) plate reader (BioTek, Winooski, VT, USA). 


\subsection{In vivo pharmacokinetic study}

All the experimental procedures were approved by the Animal Use and Care Committee of Shanghai Jiao Tong University School of Medicine. In vivo pharmacokinetics of DEB was measured in a normal rabbit aorta abdominalis, as normal vessels are typically used to study in vivo pharmacokinetics of new biomaterials $[19,20]$. Briefly, New Zealand white rabbits (SLACCAS, Shanghai, China) $(2.5-3.0 \mathrm{~kg})$ were sedated and anesthetized with ketamine $(20 \mathrm{mg} / \mathrm{kg}$, iv). The right common iliac artery was surgically exposed under sterile conditions and punctured with a $20 \mathrm{G}$ puncture needle (Terumo, Tokyo, Japan). After advancement of a 0.014-in Runthrough ${ }^{\mathrm{TM}}$ guidewire (Terumo, Tokyo, Japan) into the aorta with the tip near the aortic arch under digital subtraction angiography (INNOVA 2100, GE Healthcare, Buckinghamshire, UK), an area of the aorta abdominalis $\sim 2.5 \mathrm{~mm}$ in diameter as measured by quantitative coronary angiography (QCA) was chosen and the DEB was inflated in this area to standard $10 \mathrm{~atm}$ to $3.0 \mathrm{~mm}$ for $60 \mathrm{~s}$ with a balloon vessel ratio of 1.2:1. The arteriotomy and dermal layers were sutured after the catheter, wire, and sheath were removed. Animals were euthanized after $1 \mathrm{~h}$, or 1, 7, and 28 days after the procedure ( $n=3$ for each time point), and treated vessels (nearly $30 \mathrm{~cm}$ including the upper and lower ends of the dilated vessels) were harvested for paclitaxel measurement using a reverse phase high performance liquid chromatography (HPLC) assay. Results are expressed as the cumulative weight percent of paclitaxel released.

\subsection{Safety and efficacy study}

Five Male New Zealand white rabbits were fed normal laboratory chow for seven days. Three days before the procedure and throughout the following period, all animals received $10 \mathrm{mg}$ aspirin and $12.5 \mathrm{mg}$ clopidogrel daily (po). Arterial access was achieved with the method described above and two BMSs were implanted in the aorta abdominalis, which would damage the normal endothelial cell layer of the vessel based on previous reports [21]. A control balloon and the DEB were then randomly dilated in each of the stented vessels to $10 \mathrm{~atm}$ for $120 \mathrm{~s}$, deflated for $60 \mathrm{~s}$, and re-dilated to $10 \mathrm{~atm}$ for another $60 \mathrm{~s}$. Four weeks after the procedure animals were sacrificed with an overdose of anesthetic and the stented segments of the aorta abdominalis were harvested and immediately fixed in $10 \%$ formalin.

\subsection{Histology analysis}

The stented and balloon-dilated arterial segments, obtained after 28 days, were stained with hematoxylin and eosin (H\&E) according to published methods [21]. Neointimal thickness is obvious after 4 weeks of stent implantation in rabbit model. Samples were dehydrated in a graded series of ethanol and embedded in methylmethacrylate plastic. After polymerization, the center section of each stent was cut on a rotary microtome (EXAKT, Norderstedt, Germany) into 15-20 $\mu \mathrm{m}$ sections and stained with H\&E. An experienced pathologist blinded to the treatment groups performed all histological analyses. Ordinal data including injury and inflammation scores, neointimal thickness and area, percent lumenal stenosis, lumen area, and internal elastic membrane area were collected on each stent section according to previously reported methods [22].

\subsection{Immunohistochemistry}

Paclitaxel is a tubulin-disrupting agent that binds preferentially to $\beta$-tubulin, the basic structural unit of microtubules. Thus, paclitaxel can interfere with the normal microtubules degradation during cell division. For immunohistochemical analysis of $\beta$-tubulin, cross-sections of stented vessels were fixed in 
$10 \%$ formalin at room temperature for 1 day. Stents were then carefully removed from vessels under microscopy. Vessels were dehydrated through an upgraded ethanol series and embedded in paraffin blocks. Ultrathin sections ( $5 \mathrm{~mm}$ ) were deparaffinized, rehydrated, and incubated for $5 \mathrm{~min}$ in $3 \% \mathrm{H}_{2} \mathrm{O}_{2}$ to inhibit endogenous peroxidase activity. Masking of non-specific binding sites was performed by incubation in $5 \%$ bovine serum albumin for $30 \mathrm{~min}$. Subsequently, sections were incubated with mouse anti-rabbit $\beta$ tubulin monoclonal antibody (Abcam, Cambridge, MA, USA) diluted to a ratio of 1:1,000 overnight at $4^{\circ} \mathrm{C}$, and then incubated with biotin-conjugated rat anti-mouse secondary antibody for $30 \mathrm{~min}$ at $37^{\circ} \mathrm{C}$, and finally incubated in streptavidin-biotin complex (SABC) for $30 \mathrm{~min}$ at $37^{\circ} \mathrm{C}$. Peroxidase activity was measured after incubation with diaminobenzidine tetrahydrochloride (DAB). After washing, sections were weakly counterstained with hematoxylin. Observations and photographic records were made using a phase contrast microscope.

\subsection{Statistics}

Results are expressed as means \pm standard deviation (SD). Statistically significant differences among different treatment groups at a single time point were determined by ANOVA, followed by two-tailed Student's $t$-tests. Statistical significance was assumed for $P$ values $<0.05$.

\section{Results}

\subsection{Cytotoxicity testing}

The cytotoxicity of iopromide-coated balloon treatment was quantitatively measured via WST-1 assay. Cell viability was expressed as a fraction of viable cells and normalized to that of cells co-incubated with the bare balloon (control). Data showed that the cytotoxicity of iopromide-coated balloon was Grade 1 according to the test standard of United States Pharmacopeia (USP) XXII, as HUVEC viability in iopromide-coated balloon group was $86.4 \%$ compared with controls, suggesting reliable cytocompatibility for the DEB (Fig. 1).

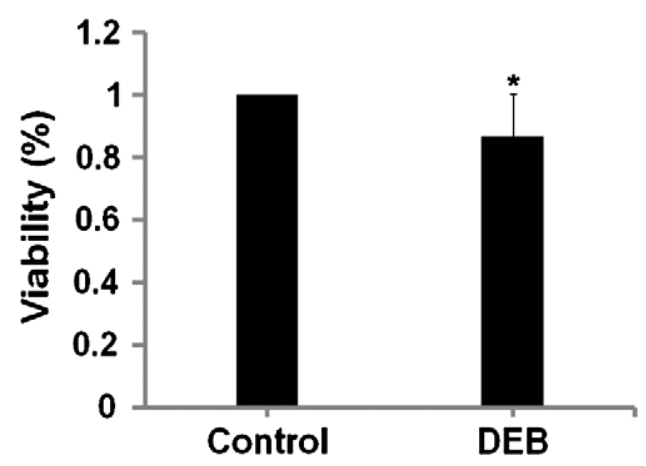

Fig. 1. WST-1 assay indicates human umbilical vein endothelial cell (HUVEC) viability after co-incubation with iopromidecoated balloon or bare balloon up to $48 \mathrm{~h}$. Data are expressed as means \pm SD of six independent experiments (ratio on control 1). ${ }^{*} P<0.05$ vs. control group. 


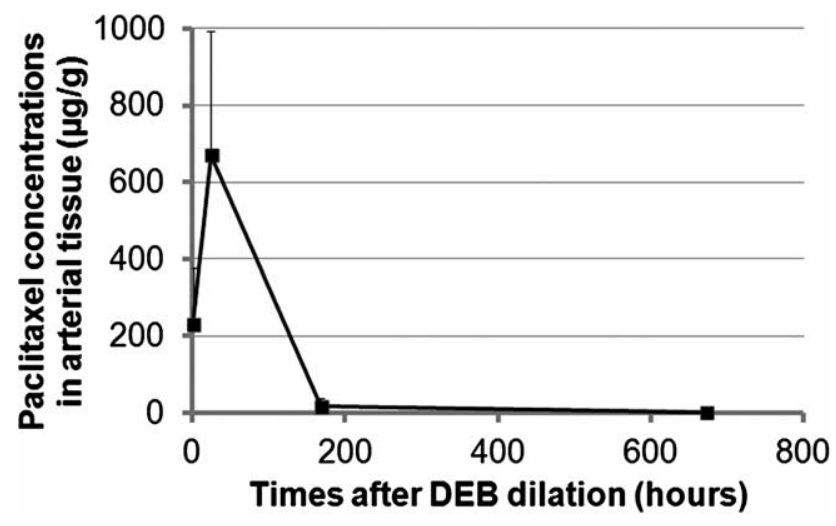

Fig. 2. Paclitaxel release kinetics of DEB in vivo.

\subsection{Release pharmacokinetics of DEB in vivo}

In vivo elution of paclitaxel from the DEB revealed that in the tissue, the cumulative diffused drug was $230.98 \pm 147.42 \mu \mathrm{g} / \mathrm{g}$ at $1 \mathrm{~h}, 670.53 \pm 323.31 \mu \mathrm{g} / \mathrm{g}$ at $1 \mathrm{day}, 15.88 \pm 21.23 \mu \mathrm{g} / \mathrm{g}$ at 7 days, and $1.1 \pm$ $1.64 \mu \mathrm{g} / \mathrm{g}$ at 28 days (Fig. 2).

\subsection{Histomorphometry and histology}

All animals underwent successful implantation of BMS and balloon dilation (Fig. 3). At 28 days, there were significant differences in luminal area, neointimal thickness, neointimal area, and percent stenotic lumen among DEB and control groups, and injury and inflammation scores were similar in both groups (Table 1; Fig. 4).

\subsection{Immunohistochemical detection of $\beta$-tubulin}

Immunohistochemically, brown nuclei were $\beta$-tubulin positive. Data show that $\beta$-tubulin was dramatically increased in the DEB group compared with the control group (arrowhead), suggesting that paclitaxel was involved in anti-restenosis effects in the DEB group (Fig. 5).

\section{Discussion}

DES cannot completely reduce restenosis after PTA and ameliorate the need for revascularization; thus, the risk of stent thrombosis remains [23,24]. Also, longer dual antiplatelet therapy of usually 12 months is needed compared to only one month for BMS [25]. Thus, BMS is preferred for $20-30 \%$ of patients [26]. DEB was therefore designed to integrate advantages of bare balloon treatment and DES. We found that a novel iopromide based paclitaxel-eluting balloon could better reduce neointimal hyperplasia in rabbit aorta abdominalis compared with bare balloon therapy.

The drug of choice for local delivery should be optimally lipophilic, highly absorbed or rapidly taken up by the intima to compensate for the short contact time, as well as a high retention rate, and sustained 


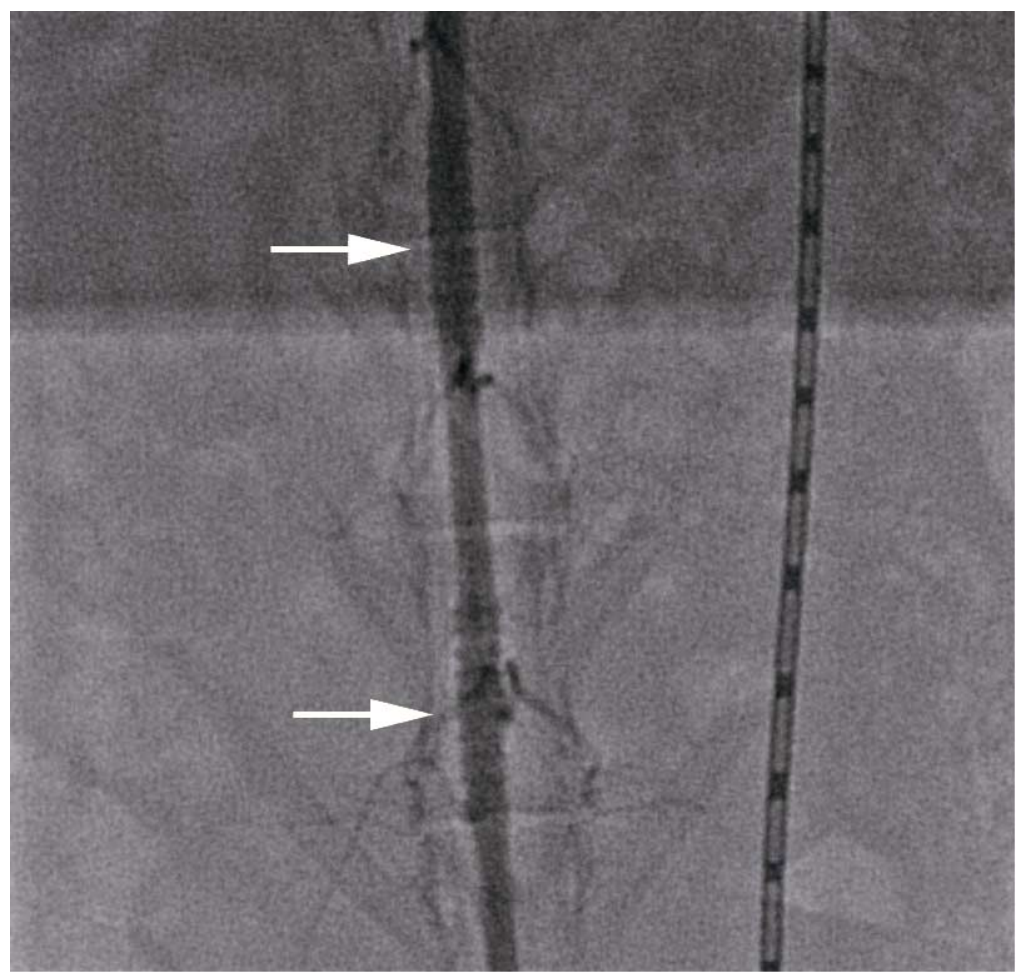

Fig. 3. Representative images of aorta abdominalis assessed by angiography immediately after BMS implantation (arrowhead: BMS implantation sites). (Colors are visible in the online version of the article; http://dx.doi.org/10.3233/BME-151551.)

Table 1

Morphometric comparisons

\begin{tabular}{|c|c|c|}
\hline & DEB group & Control group \\
\hline 28 day follow-up, $n$ & 5 & 5 \\
\hline Injury score & $1.54 \pm 0.12$ & $1.52 \pm 0.15$ \\
\hline Mean LA, mm² & $8.14 \pm 0.30^{\boldsymbol{\Lambda}}$ & $6.96 \pm 0.49$ \\
\hline Mean IEM, $\mathrm{mm}^{2}$ & $8.63 \pm 0.13$ & $8.56 \pm 0.23$ \\
\hline Mean NA, mm² & $0.49 \pm 0.18^{\boldsymbol{\Lambda}}$ & $1.60 \pm 0.29$ \\
\hline Mean NT, mm & $0.04 \pm 0.01^{\boldsymbol{\Lambda}}$ & $0.09 \pm 0.01$ \\
\hline Percent area stenosis, $\%$ & $0.06 \pm 0.02^{\star}$ & $0.19 \pm 0.04$ \\
\hline Inflammation score & $0.96 \pm 0.16$ & $0.97 \pm 0.21$ \\
\hline
\end{tabular}

LA: average lumen area; IEM: average internal elastic membrane area; NA: average neointimal area; NT: average neointimal thickness; $n$ : number of sections. ${ }^{\mathbf{\Delta}}$ Compared with control group, $P<0.05$.

effects [27]. To date, paclitaxel has met these criteria. Contrast media agents (CMA) such as iopromide adhere to the coronary vessel wall for several seconds after injection, and these have been used safely and efficiently to deliver paclitaxel to the arterial wall $[28,29]$.

Paclitaxel-coated balloon (PCB) technologies have now been clinically introduced as an alternative therapy to DES and plain balloon angioplasty (PBA) for treatment of coronary in-stent restenosis (ISR) [15,30,31], de novo peripheral artery disease [32,33], and even de novo coronary lesions [34]. Some 


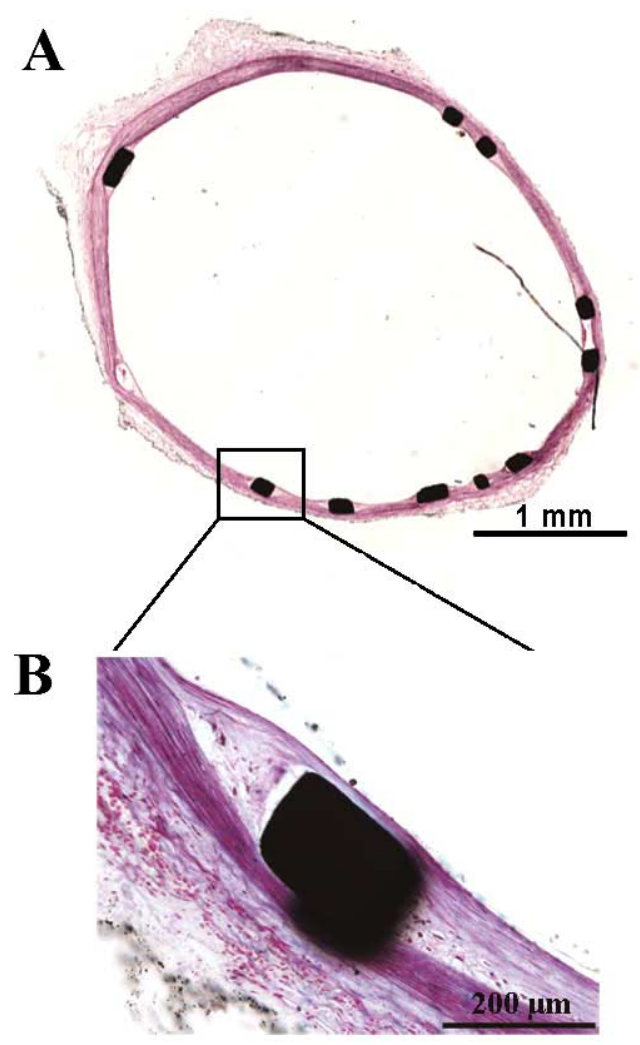

DEB

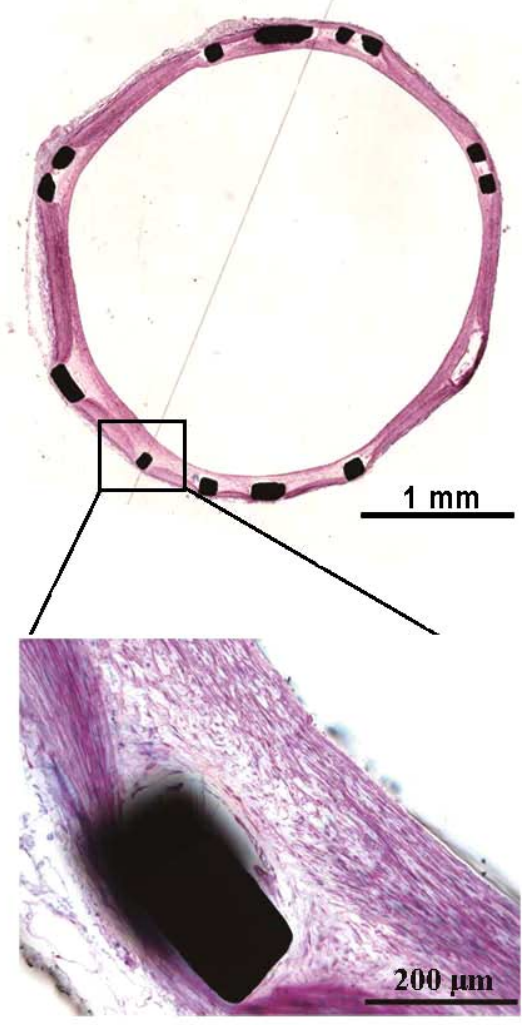

PTA

Fig. 4. Representative histological images at low-magnification (100x, upper image) and high power magnification (200x, lower image) 28 days after balloon treatment. DEB could inhibit neointimal hyperplasia in contrast with control. Sections shown are stained with hematoxylin and eosin (H\&E). (Colors are visible in the online version of the article; http://dx.doi.org/10.3233/ BME-151551.)

paclitaxel-coated balloons are also commercially available, including Paccocath ${ }^{\mathrm{TM}}$, SeQuent ${ }^{\mathrm{TM}}$, DIOR et al. [35].

It has been reported that an inflation time of $1 \mathrm{~min}$ is sufficient to allow diffusion of a high concentration of paclitaxel to the arterial wall $[14,29,36]$. In our hands, a 2-min dilation, with 1-min deflated, followed by another 1-min dilation dramatically increased paclitaxel content in the arterial tissue. Meanwhile, immunohistochemistry of paclitaxel-targeted $\beta$-tubulin revealed greater $\beta$-tubulin expression in the DEB group compared to controls.

Restenosis occurs because of vessel recoil and plaque prolapse after bare balloon angioplasty, and it can arise from neointimal hyperplasia due to smooth muscle cell migration and proliferation after stenting [37]. Moreover, stent thrombosis now is thought to chiefly occur due to a hypersensitivity reaction to the stent polymer coating [38]. Success in inhibiting neointimal proliferation has been achieved with paclitaxel which targets vascular smooth muscular cell proliferation. Although paclitaxel has been used in both DES and DEB, DES is limited by non-uniform drug delivery to the arterial and a high drug concentration at stent struts. This does not occur between the struts and at the margins [9]. Ideally, DEB should permit homogenous drug transfer to the vessel wall, avoid the use of polymers and stents and 


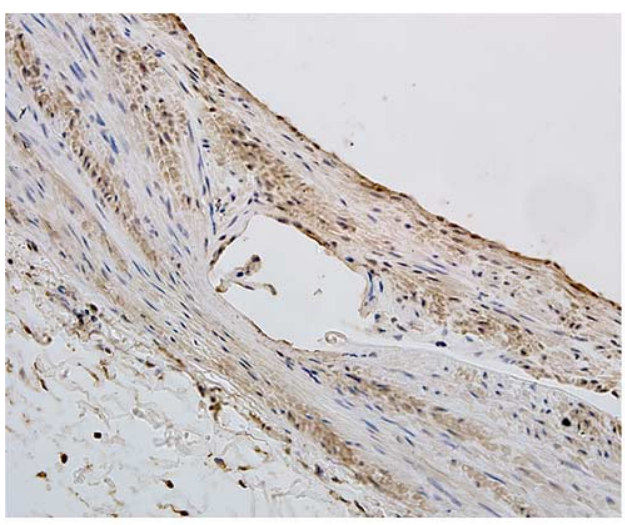

PTA

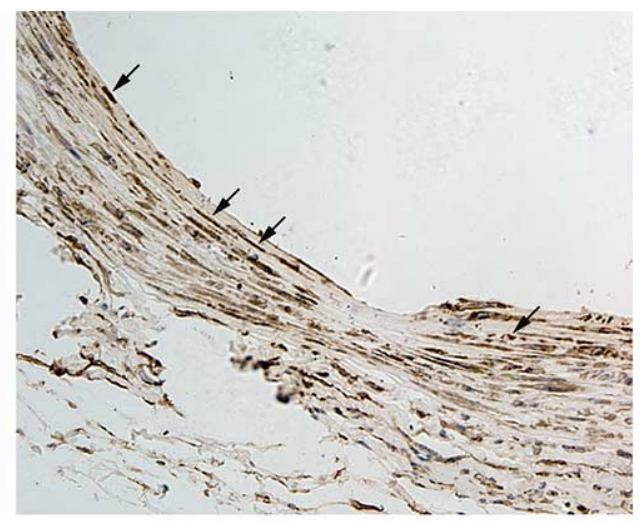

DEB

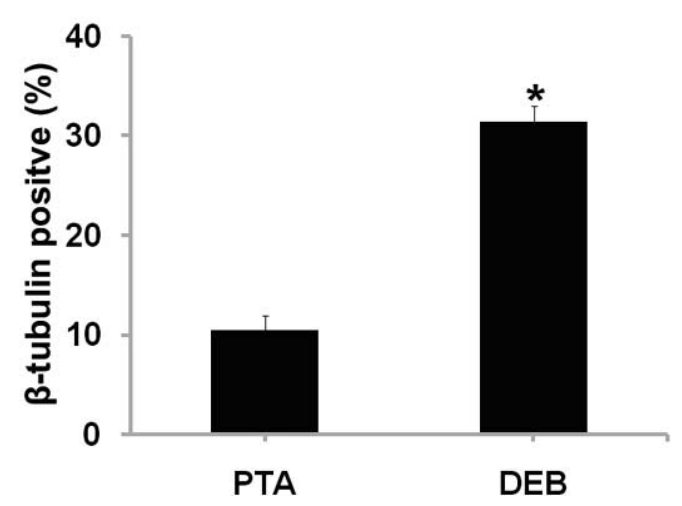

Fig. 5. $\beta$-tubulin expression in stented vascular vessels was measured via immunohistochemistry. Quantitative measurement of immunohistochemical images as a ratio of $\beta$-tubulin-positive cells indicated that $\beta$-tubulin expression was induced in the DEB group (arrowhead). All values are given as means \pm SD. $*$ significantly different compared to the control group $(P<0.05)$. (Colors are visible in the online version of the article; http://dx.doi.org/10.3233/BME-151551.)

thus obviate the need for antiplatelet therapy. Also, DEB may be used for high-risk re-stenotic lesions such as small vessel-, bifurcation-, or in-stent re-stenotic lesions.

DEB angioplasty may be a promising treatment option for ISR [31]. A recently published metaanalysis indicated that DEB was superior to bare balloon angioplasty and comparable to DES for treating DES restenosis [39]. However, DEB does not appear to be superior to current standard therapies (BMS or DES) for treating de novo coronary lesions, but may be better than BMS alone [34]. Additional and ongoing trials may help to conclude this matter [40].

\section{Conclusions}

Our results revealed a comparable efficacy profile and more favorable vascular healing response of this novel DEB. This at least partially indicate the further study of the novel DEB in treating high-risk re-stenotic lesions and pre-treatment of lesions preferred to receive BMS thus making complementary advantages. 


\section{Acknowledgements}

This work was supported by the Science and Technology Commission of Shanghai Municipality (grant 10441901102 and 12441903200), and was partly supported by the National Natural Science Foundation of China (81300178 and 81370401).

\section{References}

[1] R. Ross, Atherosclerosis - An inflammatory disease, N. Engl. J. Med. 340(2) (1999), 115-126.

[2] M.C. Morice, P.W. Serruys, J.E. Sousa, J. Fajadet, E. Ban Hayashi, M. Perin et al., A randomized comparison of a sirolimus-eluting stent with a standard stent for coronary revascularization, N. Engl. J. Med. 346(23) (2002), 1773-1780.

[3] H.J. Rapold, P.R. David, P. Guiteras Val, A.L. Mata, P.A. Crean and M.G. Bourassa, Restenosis and its determinants in first and repeat coronary angioplasty, Eur. Heart J. 8(6) (1987), 575-586.

[4] P.V. Serruys, P. de Jaegere, F. Kiemeneij, C. Macaya, W. Rutsch, G. Heyndrickx et al., A comparison of balloonexpandable-stent implantation with balloon angioplasty in patients with coronary artery disease. Benestent study group, N. Engl. J. Med. 331(8) (1994), 489-495.

[5] M.R. Bennett, In-stent stenosis: Pathology and implications for the development of drug eluting stents, Heart 89(2) (2003), $218-224$.

[6] S.O. Marx, T. Jayaraman, L.O. Go and A.R. Marks, Rapamycin-Fkbp inhibits cell cycle regulators of proliferation in vascular smooth muscle cells, Circ. Res. 76(3) (1995), 412-417.

[7] D.I. Axel, W. Kunert, C. Goggelmann, M. Oberhoff, C. Herdeg, A. Kuttner et al., Paclitaxel inhibits arterial smooth muscle cell proliferation and migration in vitro and in vivo using local drug delivery, Circulation 96(2) (1997), 636-645.

[8] B.D. Gogas, H.M. Garcia-Garcia, Y. Onuma, T. Muramatsu, V. Farooq, C.V. Bourantas et al., Edge vascular response after percutaneous coronary intervention: An intracoronary ultrasound and optical coherence tomography appraisal: From radioactive platforms to first- and second-generation drug-eluting stents and bioresorbable scaffolds, JACC Cardiovasc. Interv. 6(3) (2013), 211-221.

[9] J.W. Moses, M.B. Leon, J.J. Popma, P.J. Fitzgerald, D.R. Holmes, C. O'Shaughnessy et al., Sirolimus-eluting stents versus standard stents in patients with stenosis in a native coronary artery, N. Engl. J. Med. 349(14) (2003), 1315-1323.

[10] G.W. Stone, S.G. Ellis, L. Cannon, J.T. Mann, J.D. Greenberg, D. Spriggs et al., Comparison of a polymer-based paclitaxel-eluting stent with a bare metal stent in patients with complex coronary artery disease: A randomized controlled trial, JAMA J. Am. Med. Assoc. 294(10) (2005), 1215-1223.

[11] F. Giannini, C. Naim, C. Costopoulos, A. Latib and C.A. Drug-Coated, Balloons in interventional cardiology, Expert Rev. Cardiovasc. Ther. 11(10) (2013), 1379-1391.

[12] A. Posa, R. Hemetsberger, O. Petnehazy, Z. Petrasi, M. Testor, D. Glogar et al., Attainment of local drug delivery with paclitaxel-eluting balloon in porcine coronary arteries, Coron. Artery Dis. 19(4) (2008), 243-247.

[13] B. Cremers, M. Biedermann, D. Mahnkopf, M. Bohm and B. Scheller, Comparison of two different paclitaxel-coated balloon catheters in the porcine coronary restenosis model, Clin. Res. Cardiol. 98(5) (2009), 325-330.

[14] B. Scheller, C. Hehrlein, W. Bocksch, W. Rutsch, D. Haghi, U. Dietz et al., Treatment of coronary in-stent restenosis with a paclitaxel-coated balloon catheter, N. Engl. J. Med. 355(20) (2006), 2113-2124.

[15] M. Unverdorben, C. Vallbracht, B. Cremers, H. Heuer, C. Hengstenberg, C. Maikowski et al., Paclitaxel-coated balloon catheter versus paclitaxel-coated stent for the treatment of coronary in-stent restenosis, Circulation 119(23) (2009), 29862994.

[16] M. Werk, S. Langner, B. Reinkensmeier, H.F. Boettcher, G. Tepe, U. Dietz et al., Inhibition of restenosis in femoropopliteal arteries: Paclitaxel-coated versus uncoated balloon: Femoral paclitaxel randomized pilot trial, Circulation 118(13) (2008), 1358-1365.

[17] B. Cortese, A. Micheli, A. Picchi, A. Coppolaro, L. Bandinelli, S. Severi et al., Paclitaxel-coated balloon versus drugeluting stent during pci of small coronary vessels a prospective randomised clinical trial. The piccoleto study, Heart 96(16) (2010), 1291-1296.

[18] A. Latib, A. Colombo, F. Castriota, A. Micari, A. Cremonesi, F. De Felice et al., A randomized multicenter study comparing a paclitaxel drug-eluting balloon with a paclitaxel-eluting stent in small coronary vessels: The bello (balloon elution and late loss optimization) study, J. Am. Coll. Cardiol. 60(24) (2012), 2473-2480.

[19] J.F. Granada, M. Stenoien, P.P. Buszman, A. Tellez, D. Langanki, G.L. Kaluza et al., Mechanisms of tissue uptake and retention of paclitaxel-coated balloons: Impact on neointimal proliferation and healing, Open Heart 1(1) (2014), e000117.

[20] B. Tesfamariam, Drug release kinetics from stent device-based delivery systems, J. Cardiovasc. Pharmacol. 51(2) (2008), $118-125$. 
[21] L. Zhang, J. Zhu, R. Du, Z. Zhu, J. Zhang, W. Han et al., Effect of recombinant human SDF-1a on re-endothelialization after sirolimus-eluting stent implantation in rabbit aorta abdominalis, Life Sci. 89(25-26) (2011), 926-930.

[22] J.Z. Zhu, X.W. Xiong, R. Du, Y.J. Jing, Y. Ying, X.M. Fan et al., Hemocompatibility of drug-eluting coronary stents coated with sulfonated poly (styrene-block-isobutylene-block-styrene), Biomaterials 33(33) (2012), 8204-8212.

[23] E. Camenzind, P.G. Steg and W.W. Stent, Thrombosis late after implantation of first-generation drug-eluting stents: A cause for concern, Circulation 115(11) (2007), 1440-1455, discussion 55.

[24] J. Daemen, P. Wenaweser, K. Tsuchida, L. Abrecht, S. Vaina, C. Morger et al., Early and late coronary stent thrombosis of sirolimus-eluting and paclitaxel-eluting stents in routine clinical practice: Data from a large two-institutional cohort study, Lancet 369(9562) (2007), 667-678.

[25] P. Meier and A. Timmis, Almanac interventional cardiology: The national society journals present selected research that has driven recent advances in clinical cardiology, Heart 98(23) (2012), 1701-1709.

[26] A.P. Amin, J.A. Spertus, D.J. Cohen, A. Chhatriwalla, K.F. Kennedy, K. Vilain et al., Use of drug-eluting stents as a function of predicted benefit: Clinical and economic implications of current practice, Arch. Intern. Med. 172(15) (2012), $1145-1452$.

[27] E. Camenzind, W.H. Bakker, A. Reijs, I.M. van Geijlswijk, E. Boersma, M.J. Kutryk et al., Site-specific intracoronary heparin delivery in humans after balloon angioplasty, A Radioisotopic Assessment of Regional Pharmacokinetics, Circulation 96(1) (1997), 154-165.

[28] C. Herdeg, M. Oberhoff, A. Baumbach, A. Blattner, D.I. Axel, S. Schroder et al., Local paclitaxel delivery for the prevention of restenosis: Biological effects and efficacy in vivo, J. Am. Coll. Cardiol. 35(7) (2000), 1969-1976.

[29] U. Speck, B. Scheller, C. Abramjuk, S. Grossmann, D. Mahnkopf and O. Simon, Inhibition of restenosis in stented porcine coronary arteries: Uptake of paclitaxel from angiographic contrast media, Invest. Radiol. 39(3) (2004), 182-186.

[30] W.A. Gray and J.F. Granada, Drug-coated balloons for the prevention of vascular restenosis, Circulation 121(24) (2010), 2672-2680.

[31] A. Indermuehle, R. Bahl, A.J. Lansky, G.M. Froehlich, G. Knapp, A. Timmis et al., Drug-eluting balloon angioplasty for in-stent restenosis: A systematic review and meta-analysis of randomised controlled trials, Heart 99(5) (2013), 327-333.

[32] G. Tepe, T. Zeller, T. Albrecht, S. Heller, U. Schwarzwalder, J.P. Beregi et al., Local delivery of paclitaxel to inhibit restenosis during angioplasty of the leg, N. Engl. J. Med. 358(7) (2008), 689-699.

[33] B. Schnorr and A.T. Drug-Coated, Balloons and their place in treating peripheral arterial disease, Expert Rev. Med. Devices 10(1) (2013), 105-114.

[34] G.M. Frohlich, A.J. Lansky, D.T. Ko, O. Archangelidi, R. De Palma, A. Timmis et al., Drug eluting balloons for de novo coronary lesions - A systematic review and meta-analysis, BMC Med. 11(123) (2013). doi:10.1186/1741-7015-11-123.

[35] A. De Labriolle, R. Pakala, L. Bonello, G. Lemesle, M. Scheinowitz and R. Waksman, Paclitaxel-eluting balloon from bench to bed, Catheter Cardiovasc. Interv. 73(5) (2009), 643-652.

[36] B. Scheller, U. Speck, C. Abramjuk, U. Bernhardt, M. Bohm and N.G. Paclitaxel, Balloon coating a novel method for prevention and therapy of restenosis, Circulation 110(7) (2004), 810-814.

[37] E. Guerra, R.A. Byrne and A. Kastrati, Pharmacological inhibition of coronary restenosis: Systemic and local approaches, Expert Opin. Pharmacother. 15(15) (2014), 2155-2171.

[38] T.F. Luscher, J. Steffel, F.R. Eberli, M. Joner, G. Nakazawa, F.C. Tanner et al., Drug-eluting stent and coronary thrombosis: Biological mechanisms and clinical implications, Circulation 115(8) (2007), 1051-1058.

[39] W. Mamuti, A. Jiamali, F. Rao, W. Zhang, X. Pei, A. Ablimit et al., Drug-coated balloon angioplasty for drug-eluting stent restenosis: Insight from randomized controlled trials, Ann. Med. 46(8) (2014), 679-683.

[40] S.D. Park, C.H. Yoon, I.Y. Oh, J.W. Suh, Y.S. Cho, T.J. Youn et al., Comparison of a drug-eluting balloon first and then bare metal stent with a drug-eluting stent for treatment of de novo lesions: Study protocol of a randomized controlled trial, Trials 14(38) (2013). doi:10.1186/1745-6215-14-38. 